\title{
$V_{T H}$ subthreshold hysteresis technology and temperature dependence in commercial 4H-SiC MOSFETs
}

\author{
Besar Asllani ${ }^{\mathrm{a},{ }^{*}}$, Asad Fayyaz ${ }^{\mathrm{a}}$, Alberto Castellazzi ${ }^{\mathrm{a}}$, Hervé Morel $^{\mathrm{b}}$, Dominique \\ Planson ${ }^{\mathrm{b}}$ \\ ${ }^{a}$ PEMC Group, University of Nottingham, Nottingham, United Kingdom \\ ${ }^{\mathrm{b}}$ Univ Lyon, INSA Lyon, Univ. Claude Bernard Lyon 1, Ecole Centrale Lyon, CNRS, Ampère, F-69621, France
}

\begin{abstract}
$V_{T H}$ subthreshold hysteresis measured in commercially available 4H-SiC MOSFET is more pronounced in trench than in planar ones. All planar devices from different manufacturers exhibit an inverse temperature dependence, with the hysteresis amplitude reducing as the temperature increases, whereas all trench devices from different manufacturers exhibit the opposite behaviour. A physical interpretation is proposed, based on experimental evidence, which demonstrates that temperature dependence of the $V_{T H}$ subthreshold hysteresis is related to the technology. The findings are relevant to the ongoing discussion on $\mathrm{SiC}$ bespoke validation standards development and contribute important new insight.
\end{abstract}

\section{* Corresponding author}

besar.asllani@gmail.com

Tel: +33(0) 613120624

\section{Introduction}

Discrete SiC MOSFETs ranging from $600 \mathrm{~V}$ to $1700 \mathrm{~V}$ have been commercialised for several years and soon $3.3 \mathrm{kV}$ counterparts will be available. Before marketing, manufacturers have tested their devices in order to comply with the existing reliability standards. Nevertheless, some concerns persist since standard tests have been designed for Si technology and may not explore all of the reliability issues that exist in $\mathrm{SiC}$ devices. In particular, recent research has gained interest on threshold voltage instability of $\mathrm{SiC}$ MOSFETs [1-7]. It is important to clarify the different types of instability, their origin and of course their impact on long term reliability.

$V_{T H}$ instabilities can be categorised into two main groups:

- $\quad$ Permanent drift (NBTI, PBTI)

- $\quad$ Recoverable shift $\left(\mathrm{V}_{\mathrm{TH}}\right.$ Hysteresis)

This paper focuses in the fully recoverable $V_{T H}$ subthreshold hysteresis. Comparison amongst commercially available planar and trench $4 \mathrm{H}-\mathrm{SiC}$ MOSFETs has been carried out in order to discover any influence of the structure and technology of the device on this phenomenon.

\section{Theoretical Background}

The $V_{T H}$ subthreshold Hysteresis is defined as a recoverable increase of the subthreshold leakage current, caused by a reduction of the threshold voltage when a negative gate bias is applied at the OFF-state. Above the threshold, the hysteresis is considered to be erased and the drain current to be recovered to its characteristic value. In physical terms, it is defined as the difference between the $V_{G S} U P$, voltage of the upsweep trace, and $V_{G S}{ }^{D O W N}$, voltage of the down-sweep trace, both obtained at $100 \mathrm{nA}$ (eq. 1).

$$
\Delta V_{T H}^{H Y S T}=V_{G S}^{U P}-V_{G S}{ }^{D O W N}
$$

It has been noticed through static characterisation that $\Delta V_{T H}^{H Y S T}$ decreases for higher level of drain current until it is completely erased when the threshold level is reached [7]. Another property of $\Delta V_{T H}^{H Y S T}$ is the dependence on the negative bias $V_{G S}$ Start. Depending 
on the $V_{G S}^{\text {Start }}$, three noticeable domains can be defined as follows:

- No hysteresis

- Linear growth

- Saturation

These domains are related to the position of the Fermi Level during device operation, since by modifying its position, not only the concentration of electron and holes is changed at the semiconductor side, but also the quantity of charge (electrons and holes) captured/released from the oxide and interface traps. According to the classical theory of semiconductors
[8], the simplified behaviour of a MOSFET having an oxide containing both donor and acceptor traps is shown in figure 1 . The band diagram is represented for four possible states and traps are considered as charged or neutral depending on the Fermi level:

- $\quad$ Depletion (no bias)

- $\quad$ Flat band $\left(\mathrm{V}_{\mathrm{G}}<0 \mathrm{~V}\right)$

- Accumulation $\left(\mathrm{V}_{\mathrm{G}}<<0 \mathrm{~V}\right)$

- Inversion $\left(\mathrm{V}_{\mathrm{G}}>0 \mathrm{~V}\right)$

To satisfy the flat band condition, the Fermi level has to be shifted down by applying a negative voltage equal to $V_{F B}$, which is given by equation 2 .

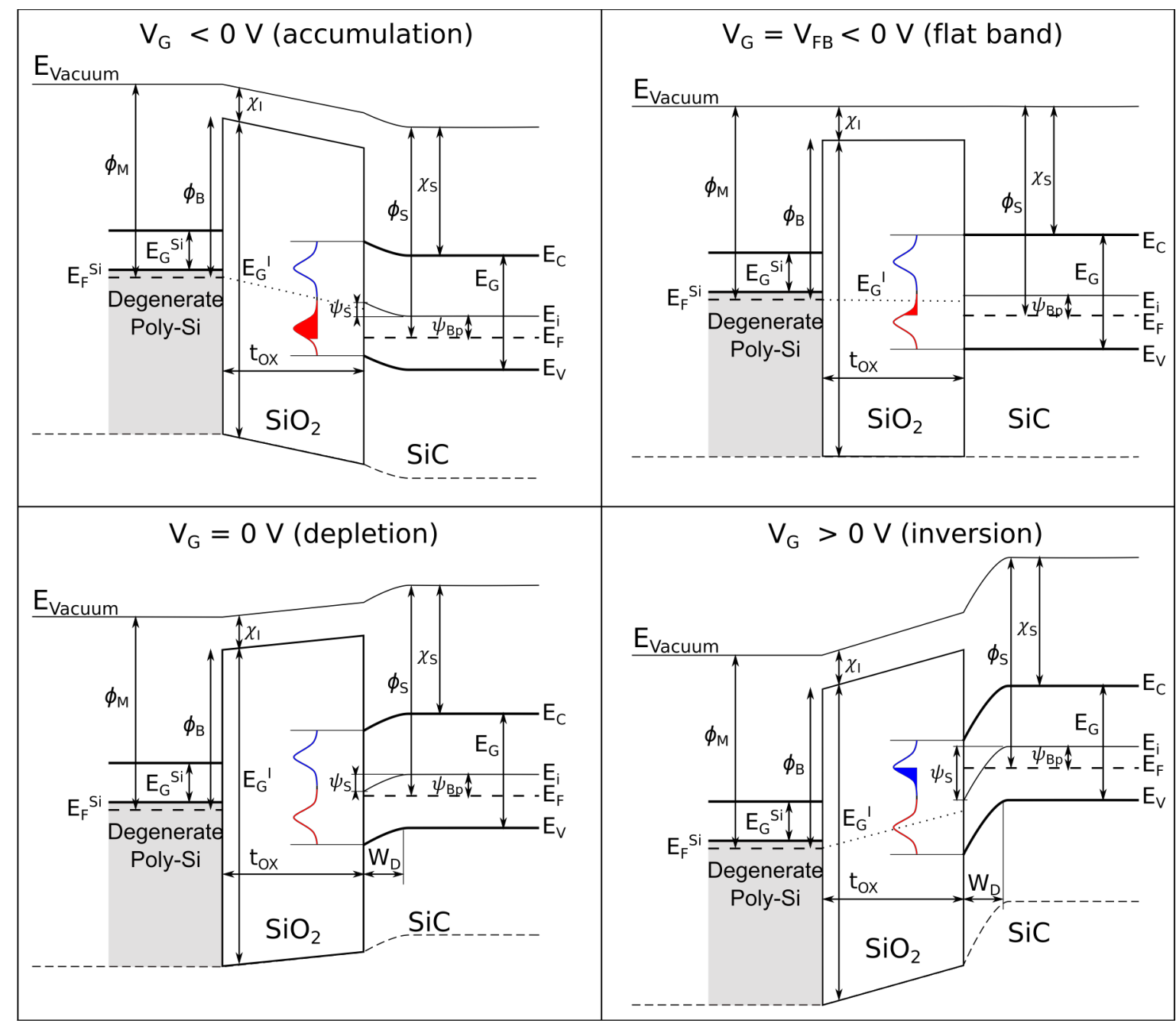

Fig. 1. The representation of the band diagram of MOSFET taking into account the workfunction difference between degenerate poly-Si gate and $\mathrm{SiC}$ and containing Gaussian distributions of both donor (red, lower part of the gap) and acceptor (blue, upper part of the gap) traps in the oxide near the interface. Mobile ions and fixed charge haven't been taken into account. The same diagram applies to planar MOSFET but instead of degenerate poly-Si metal gate is used. 


$$
V_{F B}=\phi_{M S}-\left(Q_{F}+Q_{M}+Q_{I D}\right) / C_{O X}
$$

In this equation, $\phi_{M S}$ is the difference in workfunction between the degenerate poly-Si or metal gate and the semiconductor, $Q_{F}$ is the fixed charge in the oxide, which has a great impact on the flat band voltage, $Q_{M}$ is the charge of the mobile ions in the oxide, which are neglected since clean rooms standards allow very low concentration of (sodium, potassium, lithium, etc.) contaminants [7,9], $Q_{I D}$ is the charge trapped at the interface and $C_{O X}$ is the capacitance of the oxide. The interface states are created in different ways:

- the semiconductor discontinuity [10-11]

- $\quad$ semiconductor traps [12]

- $\quad$ oxide traps near the interface [13-14]

By putting the device in the accumulation regime, the donor traps in the oxide are filled at a rate defined by their energy level and capture time constant. When the bias is applied for a long period or at a very energetic level, all of the hole traps at the interface border are filled and the $\Delta V_{T H}{ }^{H Y S T}$ saturation level is reached. In the up-sweep, the device goes from accumulation to depletion and then to inversion. During this process, small increments of gate bias are applied and the trapped holes are slowly released, meaning that the $V_{T H}$ of the device is slightly lower than expected since electrons in the bulk are attracted by the remaining trapped holes. The dependence of $V_{T H}$ on $V_{F B}$ and thus on the related traps is defined in (3),

$$
V_{T H}=V_{F B}+2 \psi_{B}+\sqrt{ }\left(4 \varepsilon_{S} q N_{A} \psi_{B}\right) / C_{O X}
$$

The maximum value of hysteresis that can be experienced in SiC MOSFETs has been calculated [6] with equation (4).

$$
\Delta V_{T H}^{H Y S T}=Q_{I D} / C_{O X}=q D_{I T} \Delta E_{I T} t_{O X} / \varepsilon_{r} \varepsilon_{0}
$$

It can range between $1.07 \mathrm{~V}$ and $10.7 \mathrm{~V}$, depending on the Density of interface and oxide traps $\left(D_{I T}=10^{11}\right.$ $\left.-10^{12} \mathrm{eV} / \mathrm{cm}^{2}\right)$ and the distribution of their energy levels $\left(\Delta E_{I T}=3.3 \mathrm{eV}\right)$ for a $70 \mathrm{~nm}$ thick oxide $\left(t_{O X}\right)$. These values are high enough to raise reliability concerns, since undesired switching can happen and as a result power electronics standards are not met.

\section{Tested devices and experimental setup}

Devices from four different manufacturers were tested. Besides manufacturer 4, which has a proprietary packaging, all the other manufacturers used TO-247 packaging. Table 1 shows the devices measured by manufacturer, rating and structure.

\begin{tabular}{llllll}
\multicolumn{6}{l}{ Table 1 - Tested devices } \\
\hline$\#$ & Rating & $V_{F B}$ & $V_{T H}$ & Struc. & Qt. \\
\hline 1 & $1.2 \mathrm{kV}-19 \mathrm{~A}$ & $-7 \mathrm{~V}$ & $3 \mathrm{~V}$ & planar & 4 \\
2 & $1.2 \mathrm{kV}-20 \mathrm{~A}$ & $-8 \mathrm{~V}$ & $3 \mathrm{~V}$ & planar & 4 \\
3 & $1.2 \mathrm{kV}-17 \mathrm{~A}$ & $-13 \mathrm{~V}$ & $4 \mathrm{~V}$ & trench & 4 \\
$4^{*}$ & $1.2 \mathrm{kV}-25 \mathrm{~A}$ & $-14 \mathrm{~V}$ & $8 \mathrm{~V}$ & trench & 4 \\
\hline
\end{tabular}

* Proprietary packaging

I(V) and C(V) tests have been carried out using a B1505A Power Device Analyser and an E4990A impedance analyser respectively. The temperature of the DUT has been controlled by a conventional hot plate with degree precision. For I(V) measurements, $V_{D S}$ has been kept constant at $1 \mathrm{~V}$, while $V_{G S}$ is swept up and down from a starting voltage of $V_{G S}$ Start up to $4 \mathrm{~V}$ with a $100 \mathrm{mV}$ step. $V_{G S}{ }^{\text {Start }}$ ranges from $-20 \mathrm{~V}$ to $0 \mathrm{~V}$ with a $1 \mathrm{~V}$ step. Since the currents involved are very small (no self heating involved), the static mode was employed and a $100 \mathrm{~ms}$ delay for each step was used. All I(V) measurements were carried out after a fast $V_{G S}$ up-sweep preconditioning from $0 \mathrm{~V}$ to $10 \mathrm{~V}$ in order to erase the effects coming from previous bias [6]. The schematic view of the $\mathrm{I}(\mathrm{V})$ test setup is illustrated in figure 2 .

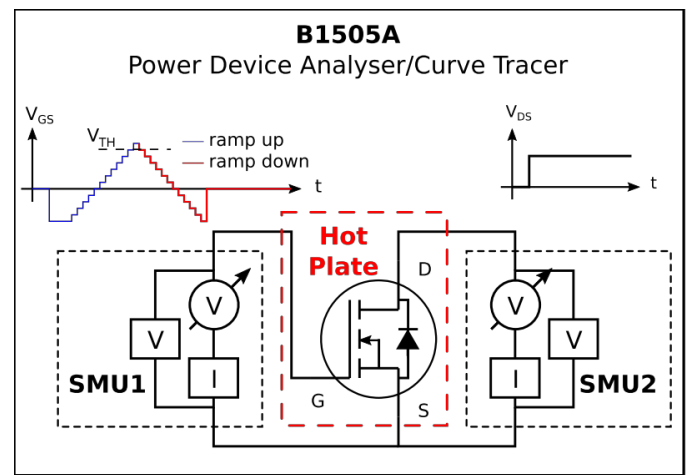

Fig. 2. Temperature dependent $V_{T H}$ Subthresold hysteresis measurement setup.

$\mathrm{C}(\mathrm{V})$ tests were carried out in order to determine the flat band voltage $V_{F B}$, the threshold voltage $V_{T H}$ and the operating (accumulation, depletion or inversion) regime of the MOSFET. The schematic $\mathrm{C}(\mathrm{V})$ setup is shown in figure 3. Drain and Source electrodes have been shorted during the measurement and the gate voltage $V_{G}$ was swept from $-20 \mathrm{~V}$ to $10 \mathrm{~V}$, for devices 1 and 2 , from $-30 \mathrm{~V}$ to $15 \mathrm{~V}$ for device 3 
and from $-30 \mathrm{~V}$ to $30 \mathrm{~V}$ for device 4 , with a $100 \mathrm{mV}$ step. An AC signal of $25 \mathrm{mV}-1 \mathrm{MHz}$ was superposed to the DC sweep voltage in order to measure the device capacitance. Measurement have been made for several operating temperatures controlled with a regulated hot plate.

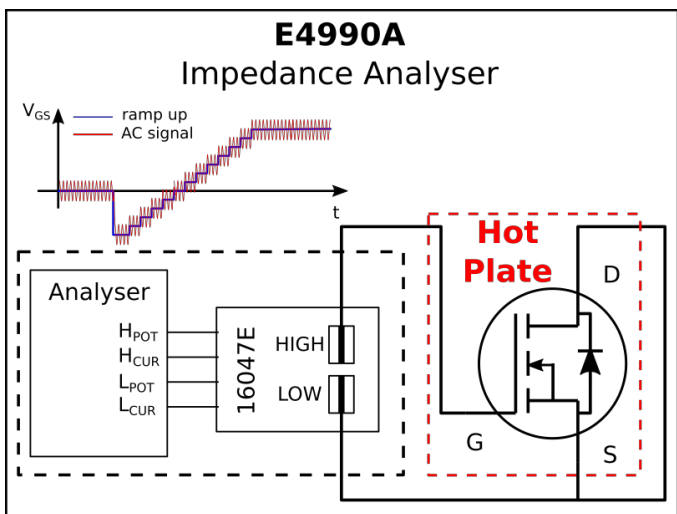

Fig. 3. Temperature dependent $\mathrm{C}(\mathrm{V})$ measurement setup for commercial $4 \mathrm{H}-\mathrm{SiC}$ MOSFETs.

\section{Results and discussion}

The measurements carried out on MOSFETs from manufacturer 1 are shown in figure 4 . The transfer characteristic differs much as $V_{G S}{ }^{\text {Start }}$ goes from $-20 \mathrm{~V}$ to $0 \mathrm{~V}$ in the up-sweep direction (dashed oval). In the down-sweep direction all the traces almost overlap (small oval), showing that independently of the previous state of the $V_{G S}$ Start , all traces are identical and that its effect has been recovered/erased. This effect has been encountered in literature as well, but details have not been given on the device's structure $[6,7]$.

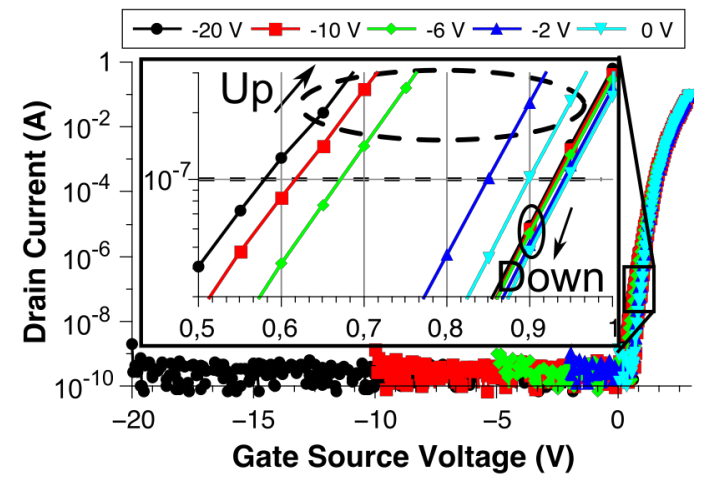

Fig. 4. $\mathrm{V}_{\mathrm{TH}}$ Subthresold hysteresis effect in a commercial $1200 \mathrm{~V} 4 \mathrm{H}-\mathrm{SiC}$ MOSFET transistor from Manufacturer 1.
The value of the $\Delta V_{T H}^{H Y S T}$ subthreshold hysteresis has been extracted on all the devices of the 4 manufacturers. As shown in figure 5, hysteresis is measurable in each of them, but it is more pronounced in trench than in planar. The manufacturing processes are different for each device since they are fabricated by different competing manufacturers. Thus, the difference between values of hysteresis measured on planar and trench devices can be considered inherent to the technology itself.

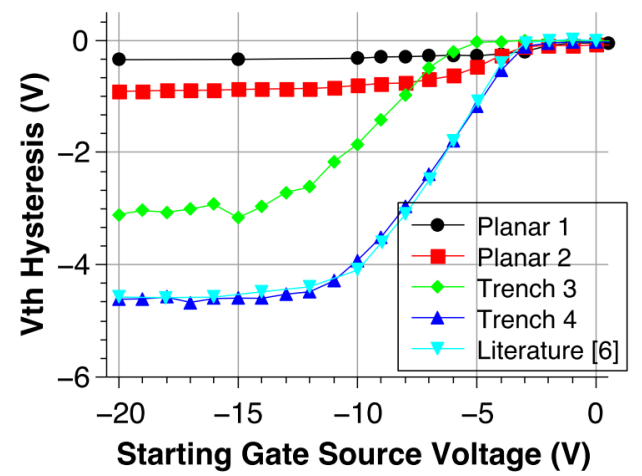

Fig. 5. $V_{T H}$ Subthresold hysteresis of all measured devices at room temperature compared to literature [6].

Referring to figure 1, when a negative bias is applied on the gate, the donor traps are filled with holes. These traps, are distributed around several energy levels in the gap and have very different relaxation time constants [15]. As a consequence, different device processing may induce different types of oxide traps, which will produce different values of hysteresis. One way to prevent a high amount of holes from being trapped in oxide near the interface is to operate the device in depletion or weak inversion mode when in off state, since fewer holes will be available near the oxide traps and the built-in voltage will create a repelling electrical field. This requires a low $V_{F B}$ though gate engineering (poly-Si doping, metal choice, channel doping and oxide fixed charge). This will also provide a low $V_{T H}$. Adjusting the design parameters (oxide thickness, channel doping, and bulk doping) can bring the $V_{T H}$ voltage to an acceptable value. As shown from the $\mathrm{C}(\mathrm{V})$ characteristics in figure 6 and table 1 , it is the case of the 4 devices, since their $V_{F B}$ is very low and the $V_{T H}$ is just a few 
volts above 0 (except for device 4 which shows a particular C(V) characteristic).

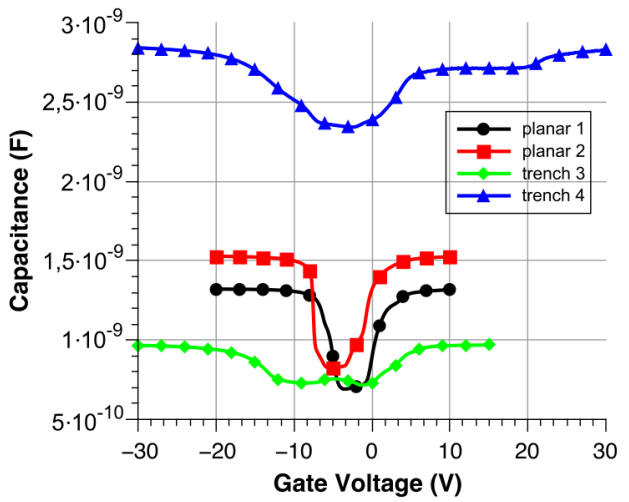

Fig. 6. Capacitance of each device measured at room temperature with a $25 \mathrm{mV}-1 \mathrm{MHz}$ oscillation.

In order to pinpoint the position of the Fermi level at the interface, the computation of the surface potential has been carried out from the $\mathrm{C}(\mathrm{V})$ characteristics at room temperature and is shown in figure 7.

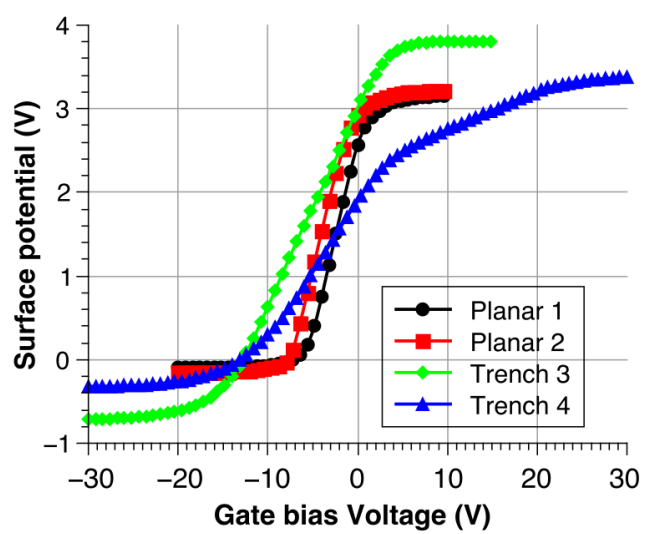

Fig. 7. Surface potential vs. gate bias extracted from $\mathrm{C}(\mathrm{V})$ measurements.

Devices 1 and 2 show a very steep variation of the surface potential and the excursion is close to the width of the band gap of the $4 \mathrm{H}-\mathrm{SiC}$, which is in conformity with the theoretical predictions. Devices 3 and 4 have a slower capacitance variation, which requires a higher gate bias to fully explore the $\mathrm{C}(\mathrm{V})$ characteristics (from accumulation to full inversion). The measurement is thus flawed because of higher gate leakage currents mostly due to hole trapping since this phenomenon occurs only when a negative bias is applied. Figure 8 shows the measured leakage current for the 4 measured devices. For positive bias, the leakage current is below the detection range of the equipment and considered very low, whereas, for negative bias, the leakage current is one magnitude higher in trench devices in the low electric field domain (dashed square). In the high electric field domain, all the devices exhibit Fowler-Nordheim tunnelling leakage current, which is often considered to be completed by trap assisted tunnelling [16]. The electrical field in the oxide can be estimated between 3 and $6 \mathrm{MVcm}^{-1}$ for a $50 \mathrm{~nm}$ thick oxide.

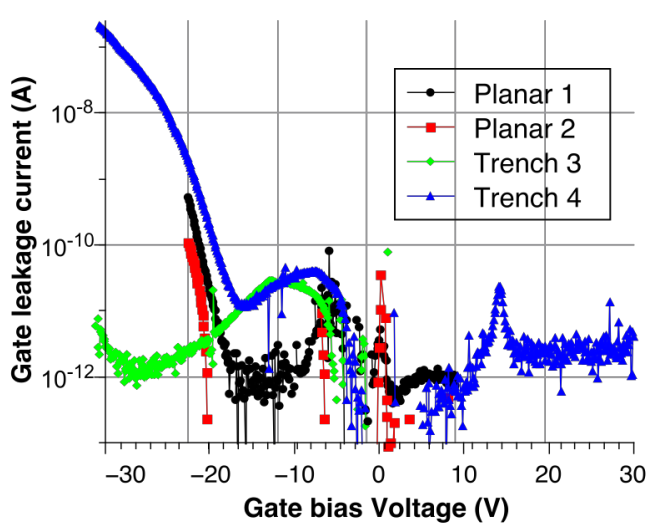

Fig. 8. Gate leakage current of the 4 measured devices.

A comparison of planar and trench technologies $\Delta V_{T H}^{S U B}$ subthreshold hysteresis for different temperatures is shown in figure 9. There is an interesting dependence on temperature and structure at the same time. Planar devices from both manufacturers exhibit a negative dependence of the hysteresis (for higher temperature hysteresis is reduced), whereas trench devices from both remaining manufacturers exhibit the opposite behaviour. These results are to be taken into account when operating trench devices, since depending on the $V_{G S}{ }^{\text {Start }}$ and temperature, the $V_{T H}{ }^{U P}$ can be only be a few tens of millivolts above $0 \mathrm{~V}$ and can considerably enhance leakage currents in the subthreshold regime. The origin of this behaviour is related to the different capture/emission time constants of the hole traps in the oxide [15]. Both these processes are accelerated when temperature is increased thus no change should be expected, but instead opposite behaviour is present for the two technologies. Since both planar devices undergo hysteresis saturation around $-7 \mathrm{~V}$ of $V_{G S}^{\text {Start }}$, the high temperature reduces the measured hysteresis because the $V_{T H}$ of the device is lowered. The trapped charge 
in saturation is almost not affected by temperature, which means that the up-sweep trace stays the same

in saturation domain whereas the down-sweep is shifted due to the $V_{T H}$ lowering. In the case of trench devices, hysteresis saturation occurs for lower negative voltage $V_{G S}$ Start. Increasing the temperature causes an acceleration of the capture of holes, which leads to a bigger trapped charge for $V_{G S}{ }^{\text {Start }}=-8 \mathrm{~V}$. The up-sweep trace is strongly affected, whereas the down-sweep is only affected by the $V_{T H}$ lowering. Since the emission time constants are generally longer compared to the capture time constants [15], the measured hysteresis is enhanced.

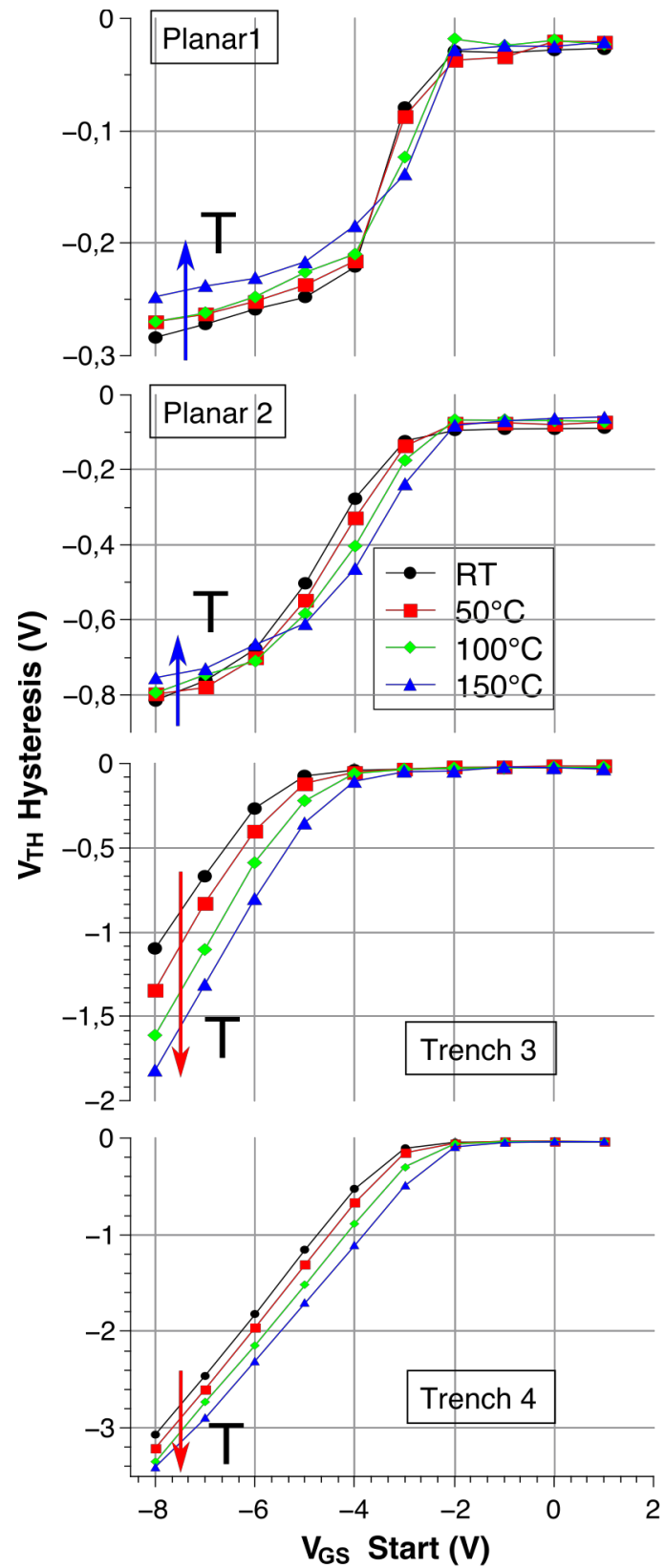

Fig. 9. $\mathrm{V}_{\mathrm{TH}}$ Subthresold hysteresis of all measured devices. Trench devices exhibit very high hysteresis which increases with temperature.

This behaviour has been normalised in figure 10 in order to show which device is more affected by temperature. Devices 1 and 2 behave very similarly, since at these conditions they operate in hysteresis saturation domain. They exhibit a very small decrease of the hysteresis. Devices 3 and 4 exhibit an increase 
of the hysteresis, but are very different. Device 3 hysteresis saturates for $V_{G S}^{\text {Start }}=-15 \mathrm{~V}$ at $-3 \mathrm{~V}$ minimum value. As a consequence, the temperature highly enhances the quantity of charge trapped in the oxide during the test at $V_{G S}{ }^{\text {Start }}=-8 \mathrm{~V}$ for different temperatures. Device 4 instead shows a higher hysteresis and its saturation appears for $V_{G S}{ }_{\text {Start }}=-11$ $\mathrm{V}$, which means that the left charge to be trapped is much smaller for $V_{G S}{ }^{\text {Start }}=-8 \mathrm{~V}$, leading to a smaller increase of the hysteresis with the effect of temperature.

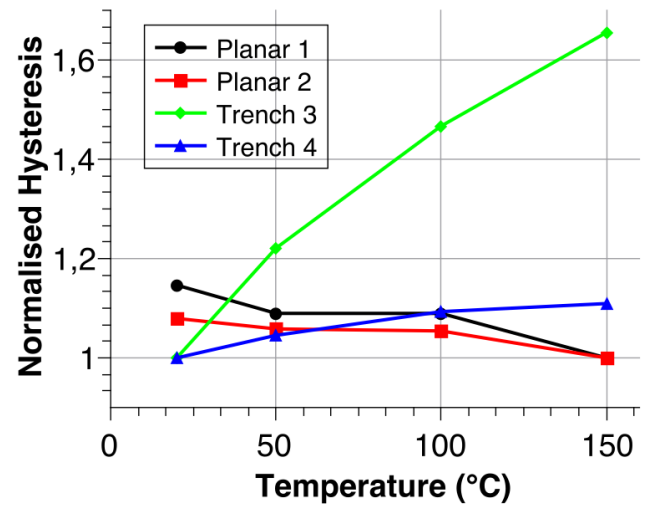

Fig. 10. Normalised $\mathrm{V}_{\mathrm{TH}}$ Subthresold hysteresis of all measured devices. Extractions are carried out for $I_{D}$ $100 \mathrm{nA}$ and $\mathrm{V}_{\mathrm{GS}}$ Start of $-8 \mathrm{~V}$.

This finding shows that planar devices must have lower density of traps $\left(D_{I T}\right)$ and the distribution of their energy levels near the valence band.

From figure 4, 7, 8 and 9, it can be stated that trench devices are affected by a higher density of oxide traps near the interface and that the distribution of their energy levels is closer to the midgap, thus more prone to hysteresis. Their energy level can be assumed closer to the midgap since the hysteresis saturation is observed for $V_{G S}{ }^{\text {Start }}$ higher than the $V_{F B}$. A schematic view of oxide border hole traps distributions in planar and trench MOSFET is shown in figure 11 .

$$
\begin{gathered}
\mathrm{V}_{\mathrm{G}}<<0 \mathrm{~V} \\
\text { (accumulation) }
\end{gathered}
$$

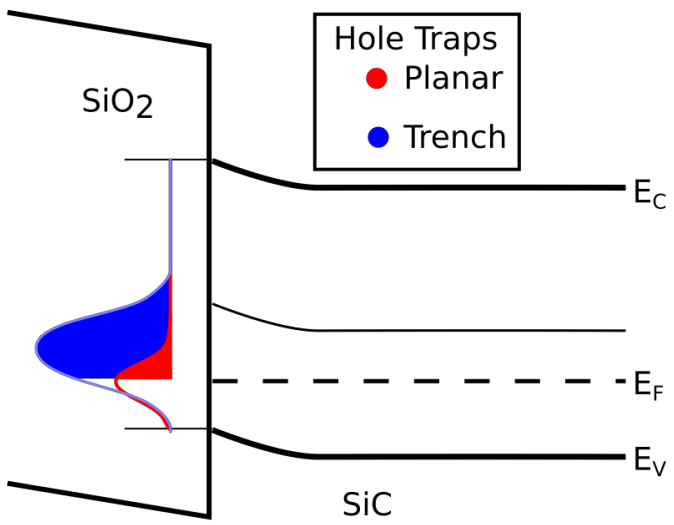

Fig. 11. Schematic illustration of the distributions of oxide border hole traps in commercially available planar and trench MOSFETs that are responsible for the $V_{T H}$ subthreshold hysteresis.

\section{Conclusions}

It has been reported that $\mathrm{V}_{\mathrm{TH}}$ subthreshold hysteresis is fully reversible and doesn't affect the device reliability [6], but there are not any studies that evaluate its evolution when devices are stressed in real application conditions. This paper shows that there is enough difference between commercial planar and trench MOSFETs to raise concerns when it comes to $V_{T H}$ subthreshold hysteresis. Further tests are needed in order to learn more on the evolution of this phenomenon in long term. A specific standard could be issued for the evaluation of this phenomenon in commercial devices.

It has been shown in this paper that trench technology is more affected by the $V_{T H}$ subthreshold hysteresis and that the phenomenon is enhanced with increasing temperature due to higher density of traps, shallower and broader distributions energy level (around midgap) compared to planar counterparts.

Dynamic characterisation of this phenomenon has been reported [17] and shows presence of hysteresis beyond the threshold voltage. Double pulse measurements with different negative OFF-state $V_{G S}$ have been reported [18] as well and show that the hysteresis persist beyond the hysteresis during turn ON but don't seem to degrade the device's performance. These measurements might shed some light on the enhancement of power losses due to 
higher leakage current caused by the $V_{T H}$ subthreshold hysteresis.

\section{References}

[1] A. J. Lelis, D. Habersat, G. Lopez, J. M. McGarrity, F. B. McLean, and N. Goldsman, "Bias Stress-Induced Threshold-Voltage Instability of SiC MOSFETs," Mater. Sci. Forum, vol. 527-529, no. January 2006, pp. 1317-1320, 2006.

[2] A. Castellazzi, T. Funaki, T. Kimoto, and T. Hikihara, "Thermal instability effects in SiC Power MOSFETs," Microelectron. Reliab., vol. 52, no. 9-10, pp. 24142419, 2012.

[3] M. Riccio, A. Castellazzi, G. De Falco, and A. Irace, "Experimental analysis of electro-thermal instability in SiC Power MOSFETs," in Microelectronics Reliability, 2013, vol. 53, no. 9-11, pp. 1739-1744.

[4] A. J. Lelis, R. Green, D. B. Habersat, and M. El, "Basic mechanisms of threshold-voltage instability and implications for reliability testing of SiC MOSFETs," IEEE Trans. Electron Devices, vol. 62, no. 2, pp. 316323, 2015.

[5] Y. K. Sharma, A. C. Ahyi, T. Isaacs-Smith, A. Modic, M. Park, Y. Xu, E. L. Garfunkel, S. Dhar, L. C. Feldman, and J. R. Williams, "High-mobility stable 4H-SiC MOSFETs using a thin PSG interfacial passivation layer," IEEE Electron Device Lett., vol. 34 no. 2, pp. 175-177, 2013.

[6] T. Aichinger, G. Rescher, and G. Pobegen, "Threshold voltage peculiarities and bias temperature instabilities of SiC MOSFETs," Microelectron. Reliab., vol. 80, pp. 68-78, Jan. 2018.

[7] G. Rescher, G. Pobegen, T. Aichinger, and T. Grasser, "On the subthreshold drain current sweep hysteresis of 4H-SiC nMOSFETs," Tech. Dig. - Int. Electron Devices Meet. IEDM, no. 1, p. 10.8.1-10.8.4, 2017.

[8] S. M. Sze and K. K. Ng, Physics of Semiconductor Devices, vol. 2007.

[9] A. Chanthaphan, "Study on Bias-temperature Instability in 4H-SiC Metal-oxide-semiconductor Devices," 2014.

[10] I. Tamm, "Über eine mögliche Art der Elektronenbindung an Kristalloberflächen," in Selected Papers, Berlin, Heidelberg: Springer Berlin Heidelberg, 1991, pp. 91-102.

[11] W. Shockley, "On the surface states associated with a periodic potential," Phys. Rev., vol. 56, no. 4, pp. $317-$ 323, Aug. 1939.

[12] R. Castagne and A. Vapaille, "Description of the $\mathrm{Si} 02-\mathrm{Si}$ interface properties by means of very low frequency MOS capacitance measurements," Surf. Sci., vol. 28 , no. 680 , pp. 157-193, 1971.

[13] G. Y. Chung, C. C. Tin, J. R. Williams, K. McDonald, M. Di Ventra, S. T. Pantelides, L. C. Feldman, R. A. Weller, "Effect of nitric oxide annealing on the interface trap densities near the band edges in the $4 \mathrm{H}$ polytype of silicon carbide," Appl. Phys. Lett., vol. 76, no. 13, pp. 1713-1715, 2000.

[14] G. Rzepa, M. Waltl, W. Goes, B. Kaczer, J. Franco; T. Chiarella, N. Horiguchi, T. Grasser, "Complete extraction of defect bands responsible for instabilities in $\mathrm{n}$ and pFinFETs," Dig. Tech. Pap. - Symp. VLSI Technol., vol. 2016-September, pp. 208-209, 2016.

[15] T. Grasser, H. Reisinger, P. J. Wagner, F. Schanovsky, W. Goes, and B. Kaczer, "The time dependent defect spectroscopy (TDDS) for the characterization of the bias temperature instability," IEEE Int. Reliab. Phys. Symp. Proc., pp. 16-25, 2010.

[16] C. T. Yen, C.-C. Hung, H.-T. Hung, C.-Y. Lee, L.-S. Lee, Y.-F. Huang, and F.-J. Hsu, "Negative bias temperature instability of SiC MOSFET induced by interface trap assisted hole trapping," Appl. Phys. Lett., vol. 108, no. 1, 012106, 2016.

[17] C. Unger and M. Pfost, "Energy capability of SiC MOSFETs," Proc. Int. Symp. Power Semicond. Devices ICs, vol. 2016-July, pp. 275-278, 2016.

[18] D. Peters, T. Aichinger, T. Basler, G. Rescher, K. Puschkarsky, and H. Reisinger, "Investigation of threshold voltage stability of SiC MOSFETs," Proc. 30th Int. Symp. Power Semicond. Devices ICs, to be published. 